\title{
Pulmonary dysfunction in children with Cystinosis: single center study, original article
}

\author{
Dina H. Hamed* ${ }^{*}$, Radwa Mohamed Abdel Halim, Mona Mohsen El Attar, Neveen A. Soliman and \\ Hanan Mohsen Osman
}

\begin{abstract}
Background: Cystinosis is a rare autosomal recessive disorder involving lysosomal storage of the amino acid cystine due to a defect in the membrane transport protein, cystinosin. The disease results in intracellular accumulation of cystine in all organs and tissues.

Pulmonary complications targeting the respiratory system occurs commonly in adults who have not received lifelong cysteamine therapy. The respiratory insufficiency is fatal at adulthood. However, little is known about the pulmonary dysfunction in children.

The study included 15 nephropathic cystinosis children to evaluate the pulmonary complications through detailed history taking, clinical examination, chest radiograph and Pulmonary function test (Impulse oscillometry \{IOS\}).

Results: Out of the cohort of 15 cystinosis patients, 13 patients did not show symptoms suggestive of chest affection. While 1 patient had recurrent Aspiration and 1 patient had history of pneumonia requiring hospital admission. All patients showed normal respiratory rate for age, normal breath sound and normal Chest $X$-ray finding apart from conical chest configuration.

IOS done showed small airway disease in 12 patients, decreased compliance in 2 patients, while only 1 patient had normal IOS.

Conclusion: Small airway affection was detected in most of cystinosis children by means of IOS in spite of the absence of chest manifestations and suggestive history which highlight the importance of respiratory assessment and follow up of cystinosis patients not only by history, and examination but most important pulmonary function tests.
\end{abstract}

Keywords: Cystinosis children, Pulmonary function tests, Small airway disease

\section{Background}

Cystinosis is a rare autosomal recessive lysosomal storage disorder caused by mutations in the CTNS gene. Main dysfunction is a defective clearance of cystine from lysosomes leading to accumulation of cystine crystals in all tissues of the body. Respiratory complications include

*Correspondence: dinahossam@yahoo.com

Pediatric Department, Faculty of Medicine, Cairo University, Cairo, Egypt respiratory failure from muscle weakness, aspiration caused by swallowing problems, and sleep apnea. Difficulty of swallowing, pulmonary dysfunction and myopathy are linked together because they are all related to muscle disease. Muscle cystine content increases with age among cystinosis patients who are not on cysteamine therapy [1]. An important unmet gap was the lack of enough information regarding the pulmonary dysfunction in children with cystinosis. 
Pharyngeal muscle involvement causes swallowing abnormality, and thoracic muscle weakness impairs pulmonary function; these are causes of death in adults with cystinosis. The frequency of myopathy is $33 \%-50 \%$, and $60 \%$ is the frequency of swallowing dysfunction [2]. Pulmonary function tests should be obtained in all patients with cystinosis when weakness in non-pulmonary muscles first becomes clinically evident and it must be done when they have dyspnea during exertion that cannot be fully explained by other causes.

Patients with orthopnea must be investigated because it may be sign of diaphragmatic weakness, this positional effect can be assessed by pulmonary function test [3].

Patients are considered to have pulmonary dysfunction if forced expiratory volume in the first second (FEV1), forced vital capacity (FVC), total lung capacity, and diffusing capacity for carbon monoxide are below $80 \%$ of predicted values [4].

Arterial blood gas measurements are not an accurate method to detect early respiratory muscle weakness. The weakness must be severe before a patient's resting minute ventilation decreases and causes arterial carbon dioxide tension increase. Also, arterial oxygen tension drops only when respiratory muscle weakness became profound, unless there are other reasons for impaired gas exchange [5].

\section{Methods}

This cross sectional study was conducted over 6 months duration from September 2018 to March 2019 on 15 patients with confirmed diagnosis of cystinosis (either newly diagnosed or previously diagnosed) by pathognomonic corneal cystine crystals in slit lamp examination, or high leukocyte cystine level. Patients were recruited from the Cystinosis Clinic, Children's Hospital. The study was approved by Research Ethical Committee of Faculty of Medicine (I-251016) and was performed in accordance with the ethical standards of Helsinki declaration. Informed consents were obtained from study subjects and/or their legal guardians before starting.

Patients' age ranged from 3 to 15 years and both genders were included.

\section{Exclusion criteria:}

Patients with cardiac impairment secondary to chest affection.

All recruited patients were subjected to all of the following:

1. Full history taking with emphasis on relevant data as follow

Date of birth, age and sex, consanguinity, family history of the same condition, history of presenting symptoms which guided the diagnosis of cystinosis,
Age of presentation, Age of diagnosis, History of chest symptoms to detect early involvement with emphasis on episodes of distress, swallowing disorder, aspiration, dysphagia, hospital admission by pneumonia, Renal replacement therapy, mode and duration.

Detailed treatment history including the details of symptomatic treatment of renal Fanconi syndrome, the cystine depleting agent with emphasis on doses, frequency and compliance of patients, and intake of immunosuppression.

\section{Clinical assessment}

Anthropometric measurements (weight, height and body mass index) and their standard deviation score assessed by growth version 2.0.

Through clinical examination with emphasis on chest examination, Inspection for conical shaped chest, respiratory rate and auscultation of the chest.

\section{Laboratory investigation}

$\square$ Assessment of renal function using blood urea nitrogen (BUN), serum creatinine concentration.

\section{$\square$ blood gases.}

Serum electrolytes including sodium $(\mathrm{Na})$, potassium $(\mathrm{K})$, calcium $(\mathrm{Ca})$, phosphorous $(\mathrm{P})$ and alkaline phosphatase (ALP).

$\square$ Complete blood count.

\section{Pulmonary function tests}

All included cases have done Impulse oscillometry which is a variant of forced oscillation technique, noninvasive method which permits passive measurement of lung mechanics: resistance at $5 \mathrm{~Hz}$ (R5), resistance at $20 \mathrm{~Hz}(\mathrm{R} 20)$, reactance at $5 \mathrm{~Hz}(\mathrm{X} 5)$, resonant frequency (Fres), area of reactance (AX). It requires minimal patient cooperation and can be done easily in subjects who are unable to perform spirometry in young children below 4 years.

Only 6 patients were able to perform spirometry (FEV1, FVC, FEV1\%, forced expiratory flow (\{FEF\} FEF25, FEF50, FEF75) as it needs patients' cooperation and older age.

5. Chest X-ray

Using TOSHIBA (DRX-1824B) at radiology department, the Children's Hospital, Faculty of Medicine.

\section{Statistical analysis}

Data were coded and entered using the statistical package SPSS (Statistical Package for the Social Sciences) version 25. Data was summarized using mean, standard deviation, median, minimum and maximum in quantitative data and using frequency (count) and relative frequency (percentage) for categorical data. Comparisons between quantitative variables were done using the non-parametric Kruskal-Wallis and Mann-Whitney tests. For comparing categorical data, Chi square test was performed. 
Exact test was used instead when the expected frequency is less than 5. Correlations between quantitative variables were done using Spearman correlation coefficient. $P$-values less than 0.05 were considered as statistically significant.

\section{Results}

Fifteen nephropathic cystinosis patients were included in the study with mean age of 8.53 .

\pm-2.85 years. Males were a little more predominant as $60 \%$ of the recruited cases were males. Table 1 summarizes the demographic data of the studied population.

The leading symptoms for diagnosis was polyuria and dehydration in 12 patients ( $80 \%$ of cases) followed by rickets in 2 patients (13\%) then failure to thrive in 1 patient (7\%).

Diagnosis was confirmed by slit lamp examination in all 15 patients and by gene analysis in only 1 patient in addition to slit lamp.

Only 7 patients (47\%) were on renal replacement therapy, 3 of them had history of renal transplantation while 4 were on regular hemodialysis.

Table 1 Demographic data of the study population

\begin{tabular}{lll}
\hline Sex & Count & Percentage \\
\hline Male & 9 & $60 \%$ \\
Female & 6 & $40 \%$ \\
Positive consanguinity & 13 & $87 \%$ \\
$\begin{array}{l}\text { Similar condition in the } \\
\text { family }\end{array}$ & 8 & $53 \%$ \\
& $\begin{array}{l}\text { Mean } \pm \text { Stand- } \\
\text { ard deviation }\end{array}$ & \\
& $8.53 \pm-2.85$ & $8(3-15)$ \\
Age (years) & $0.5 \pm 0.24$ & $0.5(0.3-1)$ \\
Age of presenting (Range) \\
Age of diagnosis (years) & $3.68 \pm 2.79$ & $4(0-8)$ \\
Weight SD & $-2.48 \pm 0.75$ & $-2.91\left(-3.00_{-}-0.78\right)$ \\
Height SD & $-4.44 \pm 2.31$ & $-4.32\left(-9.60_{-}-0.49\right)$ \\
\hline
\end{tabular}

Six patients (40\%) were CKD stage 5, 2 patients (13\%) were CKD stage 4, 3patients (20\%) were CKD stage 3 and 4 patients (26\%) were CKD stage 1 according to the GFR calculation.

All patients included in our study who were on hemodialysis were CKD stage 5, all patients with renal transplantation (100\%) were CKD stage 1, while distribution of CKD stage among patient not on renal replacement therapy was as follows: 3 patients (37\%) were CKD stage 3, 2 patients (25\%) were CKD stage 2, 2 patients $(20 \%)$ were CKD stage 4 and 1 patient (13\%) was CKD stage 1.

A statistically significant relation was detected between CKD stage and renal replacement therapy ( $p$ value 0.009) but, there was no statistically significant relation between Cystagon compliance and CKD staging (p value 0.170 ) as $50 \%$ of non-compliant patients were CKD stage 5 compared to $20 \%$ in the compliant group which may be due to the small number of patients and the late diagnosis and initiation of Cystagon therapy (Table 2).

There was no significant relation between CKD stage and age of the patients as well as age of diagnosis with $\mathrm{p}$ value (0.67 and 0.08$)$ respectively.

There was no significant relation between age of diagnosis of cystinosis and mode of renal replacement therapy as mean age of diagnosis of patient who were not on renal replacement therapy was $2.7 \pm 2.8$, mean age for those on regular hemodialysis was 6 year \pm 2.16 , and mean age of 3.1 years \pm 2.3 for transplanted patients.

All our 15 patients were compliant on renal tubular acidosis supportive therapy since the age of diagnosis, but only 5 patients (33\%) were compliant to Cystagon therapy due to the decrease availability and high cost. Only 3 patients were on immunosuppression post renal transplantation.

There was no significant relation between Cystagon compliance and mode of renal replacement therapy or weight or height affection with $p$ value $0.221,0.37,0.129$ respectively.

Table 2 Relation between chest symptoms and CKD stage and Cystagon compliance

\begin{tabular}{|c|c|c|c|c|c|c|c|c|}
\hline & & \multicolumn{6}{|c|}{ Chest symptoms } & \multirow[t]{3}{*}{$P$ value } \\
\hline & & \multicolumn{2}{|c|}{ No symptoms } & \multicolumn{2}{|c|}{ Aspiration } & \multicolumn{2}{|c|}{$\begin{array}{l}\text { Pneumonia and hospital } \\
\text { admission }\end{array}$} & \\
\hline & & Count & $\%$ & Count & $\%$ & Count & $\%$ & \\
\hline \multirow[t]{4}{*}{ CKD stage } & 1 & 4 & $31 \%$ & 0 & $0 \%$ & 0 & $0 \%$ & 1 \\
\hline & 3 & 3 & $23 \%$ & 0 & $0 \%$ & 0 & $0 \%$ & \\
\hline & 4 & 2 & $15 \%$ & 0 & $0 \%$ & 0 & $0 \%$ & \\
\hline & 5 & 4 & $31 \%$ & 1 & $100 \%$ & 1 & $100 \%$ & \\
\hline \multirow[t]{2}{*}{ Cystagon } & compliant & 5 & $38.5 \%$ & 0 & $0 \%$ & 0 & $0 \%$ & 1 \\
\hline & non-compliant & 8 & $61.5 \%$ & 1 & $100 \%$ & 1 & $100 \%$ & \\
\hline
\end{tabular}


$60 \%$ of compliant patients were not on renal replacement therapy compared to $50 \%$ of non-compliant patients.

There was no significant correlation between either weight or height of the patients and each of the following: age of the patients, age of diagnosis and CKD stage.

In our study, 13 patients (86\%) did not show symptoms suggestive for chest affection, while 1 patient had recurrent aspiration and another patient had history of pneumonia requiring hospital admission.

There was no significant relation between chest symptoms and Cystagon compliance or CKD staging as the patient who had aspiration symptom and the patient who had pneumonia as well as the four patients who did not show any chest symptoms were all CKD stage 5 (Table 2).

Significant relation was detected between CKD stage and chest wall shape with $p$ value 0.041 as 6 patients (60\%) with barrel shaped chest were CKD stage 5 (Table 3).

There was a statistically significant relation between calcium level and shape of chest wall but no significant relation was detected between phosphorus and Alkaline phosphatase level and chest wall shape (Table 4).

All our patients performed IOS technique showing small airway disease in 12 patients, decreased compliance in 2 patients, while 1 patient was normal.

But, only 6 patients were able to perform spirometry showing restrictive pattern in 3patients, obstructive pattern in 1 patient while 2 patients were normal.

Table 3 Relation between chest wall shape and CKD stage

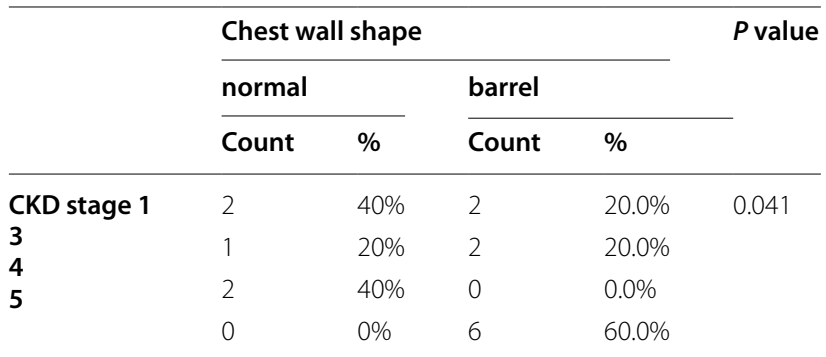

No significant relation was found between pulmonary functions either IOS (R5, R20, X5, AX) or Spirometry (FEV1, FVC, FEV1/FVC, FEF25, FEF50) and each of age, sex, duration of illness or adherence to therapy or CKD stage. Apart from significant relation between Fres and each of age and duration of illness $(P$ value $0.003,0.046$ respectively) and between FEF75 and duration of illness ( $P$ value 0.037$)$.

Also, no relation was detected between chest wall shape and each of IOS and spirometry values (Table 5).

\section{Discussion}

Cystinosis is the major cause of inherited Fanconi syndrome, and should be suspected in young children with failure to thrive and signs of renal proximal tubular damage. Nephropathic (infantile) cystinosis is the most common and the most severe clinical expression of the disease. It clinically presents with growth failure, proximal tubular Fanconi syndrome at 6-12 months of age, glomerular failure by the age of 10 years, and various nonrenal complications including a distal vacuolar myopathy, decreased pulmonary function, swallowing impairment, and deterioration of the central nervous system (CNS), endocrinopathies, vascular calcifications, retinal damage, and other ophthalmic complications [6].

One set of complications targets the respiratory system which are respiratory failure from muscle weakness, aspiration because of swallowing abnormalities, and sleep apnea.

Therefore, the current study aimed to evaluate pulmonary complications among pediatric patients with nephropathic cystinosis.

The study was conducted on 15 patients (from 15 families) with confirmed nephropathic cystinosis and included 9 males (60\%) and 6 females (40\%). Although the mean age at onset of symptoms was 0.5 years, consistent with infantile onset, yet the mean age at diagnosis was late at 3.6 years, in contrary to the recent recommendations by Nesterova and Gahl for early diagnosis and early treatment [7].

As a result of being relatively late in diagnosis and thus treatment, $40 \%$ of studied patients were CKD stage 5,

Table 4 Relation between chest wall shape and laboratory parameters

\begin{tabular}{|c|c|c|c|c|c|}
\hline & \multicolumn{4}{|c|}{ chest wall shape } & \multirow[t]{3}{*}{$P$ value } \\
\hline & \multicolumn{2}{|l|}{ normal } & \multicolumn{2}{|l|}{ barrel } & \\
\hline & Mean \pm SD & Median - range & $\begin{array}{l}\text { Mean } \pm \\
\text { SD }\end{array}$ & Median - range & \\
\hline Calcium & $10.62 \pm 0.69$ & $10.40\left(10 \_11.40\right)$ & $9.59 \pm 0.66$ & $9.50\left(9 \_10.80\right)$ & 0.040 \\
\hline phosphorus & $4.54 \pm 1.08$ & $4.10\left(3.50 \_5.80\right)$ & $4.76 \pm 1$ & $4.30\left(3.80 \_6.70\right.$ & 0.594 \\
\hline Alkaline phosphatase & $365.80 \pm 77.83$ & $333\left(295 \_481\right)$ & $330.90 \pm 197.25$ & $327(145$ _ 764) & 0.679 \\
\hline
\end{tabular}


Table 5 Relation between chest wall shape and pulmonary function tests

\begin{tabular}{|c|c|c|c|c|c|}
\hline & \multicolumn{4}{|c|}{ chest wall shape } & \multirow[t]{3}{*}{$P$ value } \\
\hline & \multicolumn{2}{|l|}{ Normal } & \multicolumn{2}{|l|}{ Barrel } & \\
\hline & Mean \pm SD & Median / range & Mean \pm SD & Median / range & \\
\hline R5 & $136.42 \pm 10.22$ & $\begin{array}{l}137.80 \\
\left(123.90 \_151.00\right)\end{array}$ & $139.89 \pm 22.39$ & $\begin{array}{l}145.05 \\
\left(102.10 \_163.20\right)\end{array}$ & 0.513 \\
\hline R20 & $132.32 \pm 21.72$ & $\begin{array}{l}129.20 \\
\left(106.30 \_165.00\right)\end{array}$ & $116.53 \pm 15.92$ & $\begin{array}{l}118.80 \\
\left(94.30 \_137.00\right)\end{array}$ & 0.206 \\
\hline $\mathrm{X} 5$ & $91.10 \pm 30.77$ & $\begin{array}{l}86.20 \\
\left(51.50 \_132.50\right)\end{array}$ & $41.04 \pm 59.55$ & $\begin{array}{l}40.00 \\
\left(-89.40 \_114.90\right)\end{array}$ & 0.099 \\
\hline Fres & $39.51 \pm 9.94$ & $\begin{array}{l}44.95 \\
\left(22.92 \_46.94\right)\end{array}$ & $38.47 \pm 16.30$ & $\begin{array}{l}39.30 \\
\left(4.11 \_58.13\right)\end{array}$ & 0.768 \\
\hline$A x$ & $4.01 \pm 2.35$ & $\begin{array}{l}3.32 \\
\left(1.60 \_7.01\right)\end{array}$ & $4.52 \pm 1.69$ & $\begin{array}{l}4.31 \\
\left(1.88 \_7.01\right)\end{array}$ & 0.594 \\
\hline FEV1 & $93.65 \pm 6.15$ & $\begin{array}{l}93.65 \\
\left(89.30 \_98 .\right)\end{array}$ & $77.38 \pm 29.01$ & $\begin{array}{l}70.60 \\
\left(53.70 \_114.60\right)\end{array}$ & 0.533 \\
\hline FVC & $90.90 \pm 1.27$ & $\begin{array}{l}90.90 \\
\left(90.00 \_91.80\right)\end{array}$ & $68.30 \pm 25.10$ & $\begin{array}{l}62.75 \\
\left(47.50 \_100.20\right)\end{array}$ & 0.533 \\
\hline FEV1\% & 107 & 107 & $113.15 \pm 2.27$ & $\begin{array}{l}113.80 \\
\left(110 \_115\right)\end{array}$ & 0.400 \\
\hline FEF25\% & $111.05 \pm 37.55$ & $\begin{array}{l}111.05 \\
\left(84.50 \_137.60\right)\end{array}$ & $90.75 \pm 49.29$ & $\begin{array}{l}76.85 \\
\left(52.80 \_156.50\right)\end{array}$ & 0.800 \\
\hline FEF50\% & $66.70 \pm 32.10$ & $\begin{array}{l}66.70 \\
\left(44.00 \_89.40\right)\end{array}$ & $73.03 \pm 25.84$ & $\begin{array}{l}62.10 \\
\left(56.80 \_111.10\right)\end{array}$ & 1.000 \\
\hline FEF75\% & $82.45 \pm 20.58$ & $\begin{array}{l}82.45 \\
\left(67.90 \_97.00\right)\end{array}$ & $60.15 \pm 12.09$ & $\begin{array}{l}56.00 \\
\left(50.70 \_77.90\right)\end{array}$ & 0.267 \\
\hline
\end{tabular}

$13 \%$ were CKD stage $4,20 \%$ were CKD stage 3 and $26 \%$ were CKD stage 1.

The age of ESRD ranged from 3 to 8 years with mean 4.5 years which is lower than that described by Starlen and his colleagues who studied 134 cystinosis on RRT and reported age of RRT at 10.8 years. This is presumably a reflection of the relatively late diagnosis coupled with inconsistent cysteamine therapy [8].

Gahl study reported that every month of treatment prior to 3 years of age, worth 14 months of preservation of renal function. This emphasizes the need for early diagnosis and early initiation of treatment to preserve renal function and prevent complications [9].

Oral cysteamine therapy was available in Egypt since June 2004 with interrupted periods due to financial constraints and lack of governmental and health insurance support. The mean dose of oral cysteamine used, which was solely supplied by charity organizations, was $35.3 \mathrm{mg} / \mathrm{kg} / \mathrm{d}$ (range $10-55 \mathrm{mg} / \mathrm{kg} / \mathrm{d}$ ).

Our study showed that $67 \%$ of our patients were non-compliant to cystagon with no significant relation between cystagon compliance, CKD stage and mode of renal replacement therapy, this may be due to small size of studied group and their young age, time gap between presenting symptom and age of diagnosis. There were no sufficient data to determine if long-term cysteamine therapy was correlated with better pulmonary function. Similar to Anikster 2001 who studied 12 adult patients who lived at least 17 years without being compliant to cysteamine therapy [10].

The clinical presentation of our patient fit the broad definition of Fanconi syndrome as the leading symptom for diagnosis was polyuria and dehydration representing $80 \%$ followed by rickets representing $13 \%$ and finally failure to thrive representing $7 \%$.

The rate of consanguineous marriage in Egypt is still high, thus autosomal recessive inherited disorders are common as positive consanguinity was detected in $87 \%$ of patients, 8 families had other affected siblings with cystinosis.

Growth was greatly affected in our patients but, height was affected more than weight as median weight was -2.9 ranging from $-3 \mathrm{SD}$ to $-0.78 \mathrm{SD}$ with while median height -4.3 ranging from $-9.6 \mathrm{SD}$ to-0.49SD. This is partly due to metabolic bone disease including active rickets and renal osteodystrophy in patients with ESRD.

Out of 15 cystinosis patients, only 3 patients received renal allograft. Anikster studied 12 adult patients with nephropathic cystinosis and 3 patients with ocular, nonnephropathic cystinosis and all the nephropathic cystinosis patients had previously received a renal allograft [10]. 
Our patients underwent a thorough pulmonary evaluation that included a clinical assessment, pulmonary function tests in the form of IOS/ Spirometry and chest $\mathrm{x}$-ray. But not all our patients were able to perform spirometry which is more sensitive in detecting restrictive lung disorder expected to be found in cystinosis patients due the young age of our studied patient (ranging from 3 to 15 years with mean of 8.6 years) and the need of patients' cooperation and revealing no significant relation between pulmonary function affection and age, sex, age of diagnosis, consanguinity, immune suppression medication or chest symptoms and chest wall shape.

Out of the 6 patients performing spirometry, 3 patients showed restrictive pattern with mean age predicted value of FEV1 and FVC were 82.8 and 75.8 respectively compared to mean FEV1 and FVC 57 and 58 respectively in an adult study including 12 nephropathic cystinosis patients (age ranged from 21 to 40 years) and detected decreased values for mean FVC, FEV1, and total lung capacity but there was no correlation between impaired pulmonary function and age, gender, or immunosuppressive medications [10]. This difference attributed to predict value to age and age difference in both studied group as cystine crystals continue accumulation in tissues including muscles as age progress.

This is the first study to evaluate the pattern of lung dysfunction in Egyptian children affected by nephropathic cystinosis. There was no available study in nephropathic cystinosis to compare IOS values with data received from our study.

Clinically all our recruited patients had normal respiratory rate for age, normal breath sound without adventitious sounds, but by chest inspection (10 patients) showed increment in AP diameter of the chest while (5 patients) showed normal chest wall. This due to rickets associated with renal disease and renal osteodystrophy proven by statistically significant relation between calcium level and chest wall shape with $p$ value 0.04 . Eighty six percent (86\%) of our studied patients did not show symptoms suggestive for chest affection, 1patients (7\%) had recurrent aspiration while another one (7\%) had pneumonia requiring hospital admission. In contrast to an adult study showing that 9 patients had no respiratory complaints, 6 complained of dyspnea at night and 2 patients died of respiratory insufficiency [10].

Chest radiograph done for our studied patients did not show any abnormality except for conical chest configuration. In contrary to Anikster study, which performed chest radiography for 11 patients showing normal lung parenchyma in 9 patients but slightly increased interstitial markings in 1patient and left base atelectasis in 1 patient. In addition, seven patients exhibited a conical chest cavity thin-section. CT scans of the chest was also performed for 11 patients. The lung parenchyma was normal in nine patients, while 1 patient exhibited a punctate density in the pleura of the right upper lobe and 1 patient showed minimal scarring of the left lower lobe. This difference can be attributed for younger age where pulmonary affection known to be a late complication of the disease [10].

Our study showed no significant relation between CKD stage or mode of renal replacement therapy and pulmonary complication which was similar to Anikster study that showed no relation of pulmonary disease with renal failure, as indicated by the normal serum creatinine values in 10 out of 12 patients [10].

Patients in our studied group did not show symptoms suggestive of myopathy in the form of dysphagia or recurrent aspiration so no EMG was performed, therefore; we lack relation between pulmonary functions and myopathy. In contrast to the adult study which proved that the extent of pulmonary dysfunction correlated directly with the severity of myopathy. The respiratory muscle seems not to be involved in the myopathic process, although recruitment of the intercostal muscles during quiet respiration was noticed. Limited EMG examination detected evidence for irritability of the biceps and intercostal muscles, typical of the primary muscle disorder seen in nephropathic cystinosis [10].

\section{Conclusion}

Finally, we concluded that small airway affection was detected in most of cystinosis children by means of IOS in spite of the absence of chest manifestations and suggestive history which highlight the importance of respiratory assessment and follow up of cystinosis patients not only by history, and examination but most important pulmonary function tests.

A major obstacle in our study was the small sample size being a rare disease as well as the difficulty in performing Spirometry by some of the patients.

\section{Abbreviations \\ IOS: Impulse oscillometry; FEV1: Forced expiratory volume in the first second; FVC: Forced vital capacity; BUN: Blood urea nitrogen; Na: Sodium; K: Potassium; Ca: Calcium; P: Phosphorous; ALP: Alkaline phosphatase; R5: Resistance at $5 \mathrm{~Hz}$; R20: Resistance at $20 \mathrm{~Hz} ; \mathrm{X} 5$ : Reactance at $5 \mathrm{~Hz}$; RF: Resonant frequency; AX: Area of reactance; CKD: Chronic kidney disease.}

\section{Acknowledgements}

We are really grateful to all the patients who participated in the study.

\section{Availability of data and material}

All raw material is available upon reasonable request.

\section{Authors' contributions}

D. H.: contributed in the selection of patients, performance and interpretation of the pulmonary function tests, drafting, final revision and approval of the manuscript. R.M.: contributed in the selection of cystinosis patients, collection of demographic and clinical data, drafting and final revision of the manuscript. 
M. M.: contributed in the study design, final revision on the interpretation of pulmonary function tests and approval of the manuscript. N.A.S.: contributed in the study design, selection of cystinosis patients, final revision and approval of the manuscript. H.M.O: contributed in the performance and interpretation of the pulmonary function tests, final revision and approval of the manuscript.

\section{Funding}

The study is self-funded.

\section{Ethics approval and consent to participate}

The study was approved by Research Ethical Committee of Faculty of Medicine, Cairo University (I-251016) and was performed in accordance with the ethical standards of Helsinki declaration. Written consents were obtained from study subjects and/or their legal guardians before starting.

\section{Consent for publication}

Not applicable.

\section{Competing interests}

No competing interest.

Received: 21 June 2021 Accepted: 10 January 2022

Published online: 07 February 2022

\section{References}

1. Gahl WA, Thoene JG, Schneider JA (2002) Cystinosis. N Engl J Med 347:111-121

2. Theodoropoulos DS, Shawker TH, Heinrichs C, GahI WA (1995) Medullary nephrocalcinosis in nephropathiccystinosis. Pediatr Nephrol 9:412-418

3. Ariceta G, Camacho JA, Fernandez-Obispo M, Fernandez-Polo A, Gamez J, Garcia-Villoria J et al (2015) Cystinosis in adult and adolescent patients: recommendations for the comprehensive care of cystinosis. Nefrologia 35:304-321

4. Sonies BC, Almajid P, Kleta R, Bernardini I, Gahl WA (2005) Swallowing dysfunction in 101 patients with nephropathic cystinosis - benefit of long-term cysteamine therapy. Medicine 84:137-146

5. Edens MA, van Son WJ, de Greef MH, Levtchenko EN, Blijham T, Wijkstra PJ. Successful treatment of respiratory dysfunction in cystinosis by nocturnal non-invasive positive pressure ventilation. Clin Nephrol 2006; 66:306-9)

6. Martijn J. Wilmer, Joost P. Schoeber, Lambertus P. van den Heuvel, 1 and Elena N. Levtchenko. Cystinosis: practical tools for diagnosis and treatment. Pediatr Nephrol. 2011 26(2): 205-215.

7. Nesterova G, Gahl WA (2013) Cystinosis: the evolution of a treatable disease. Pediatr Nephrol. 28:51-59

8. Van Stralen KJ1, Emma F, Jager KJ, Verrina E, Schaefer F, Laube GF, et al. Improvement in the renal prognosis in nephropathic cystinosis. Clin J Am Soc Nephrol 2011 Oct;6(10):2485-2491. doi: https://doi.org/10.2215/CJN. 02000311.

9. Gahl WA, 2003. Early oral cysteamine therapy for nephropathic cystinosis. Eur J Pediatr. 2003 Dec;162 Suppl 1: S38-41.

10. Anikster Y, Lacbawan F, Brantly M, Gochuico BL, Avila NA, Travis W et al (2001) Pulmonary dysfunction in adults with nephropathic cystinosis. Chest 119:394-401

\section{Publisher's Note}

Springer Nature remains neutral with regard to jurisdictional claims in published maps and institutional affiliations.

\section{Submit your manuscript to a SpringerOpen ${ }^{\circ}$ journal and benefit from:}

- Convenient online submission

- Rigorous peer review

- Open access: articles freely available online

- High visibility within the field

- Retaining the copyright to your article 